\title{
Performance cognitive de futurs professionnels de l'intervention
}

\author{
SERGE LARIVÉE* et SYLVIE NORMANDEAU**
}

\section{RÉSUMÉ}

Le niveau opératoire de 48 étudiants universitaires en sciences humaines est mesuré à l'aide d'épreuves relevant du schème de la combinatoire, de la probabilité et du contrôle des variables. Le pourcentage des étudiants atteignant les niveaux formels aux épreuves du contrôle des variables ne depasse pas 50\% alors que celui obtenu aux épreuves de probabilité et de combinatoire monte à 90\%. Les résultats sont analysés en fonction de l'effet de l'apprentissage scolaire et du contenu des tâches.

\begin{abstract}
Cognitive levels of 48 University students enrolled in humanities were assessed by piagetian tasks. Three specific formal abilities were measured: combinatory, probability, control of variables. For the control of variable tasks, the percentage of students reaching the formal levels did not exceed $50 \%$ while that of probability and combinatory tasks went up to $90 \%$. The results are analyzed according to school training effect and content of tasks.
\end{abstract}

En accord avec la théorie opératoire, le stade des opérations formelles devrai† être atteint, sinon entre 12 et 15 ans, du moins entre 15 et 20 ans (Inhelder et Piaget, 1955; Piaget, 1972; Piaget et Inhelder, 1963, 1966). Ainsi les étudiants de niveau universitaire devraient tous pouvoir aisẻment recourir à des habiletés opératoires formelles. En effet, d'une part, la scolarité pré-universitaire comprend des programmes dont les contenus (v.g. mathématique, physique, chimie) nécessitent l'utilisation des schèmes formels (combinatoire, contrôle des variables, corrélation, probabilité, proportion). D'autre part, les programmes universitaires exigent habituellement un niveau d'abstraction caractéristique de la pensée

* Ecole de psycho-éducation, Université de Montréal

** Département de psychologie, Université du Québec à Montréal 
formelle (raisonnement hypothético-déductif, logique propositionnelle, ouverture sur les possibles). Dès lors, on devrait s'attendre à retrouver chez les étudiants accédant au niveau universitaire les compétences cognitives nécessaires pour rencontrer les exigences des programmes (Barnes, 1977; Good, 1977; Lawson \& Renner, 1974; McKinnon \& Renner, 1971).

Cependant récemment un certain nombre de chercheurs (voir tableau 1) se sont préoccupés d'évaluer le niveau opératoire d'étudiants de niveau collégial et universitaire et leurs résultats présentent des pourcentages variables et parfois peu élevés d'étudiants utilisant les raisonnements opératoires formels théoriquement attendus. Les résultats présentent aussi une grande variabilité dans les pourcentages de réussite selon les types de passation (clinique versus test papiercrayon), les schèmes étudiés (proportion, probabilité, contrôle des variables...), les critères d'analyse et le nombre de niveaux opératoires identifiés.

Plus particulièrement, les résultats diffèrent sensiblement selon que le contenu des épreuves est ou non scolaire. Ainsi le pourcentage de réussite au niveau formel $\mathrm{B}$ varie entre $14 \%$ et $52.7 \%$ pour le schème du contrôle des variables, schème non appris en tant que tel à l'école. Toutefois, lorsque les schèmes évalués sont de nature scolaire, les résultats obtenus sont meilleurs: les pourcentages de réussite au niveau formel B varient entre $73.3 \%$ et $82.3 \%$ pour le schème des probabilités, entre $4.6 \%$ et $95.4 \%$ pour le schème de la combinatoire et entre $23.6 \%$ et $92.4 \%$ pour le schème des proportions. On peut penser que de telles disparités dans les résultats reflètent, soit l'influence de l'apprentissage scolaire concourant ainsi à des résultats plus élevés pour certaines tâches, soit l'influence du contenu et des intérêts sur la réussite. Le contenu logico-mathématique de certaines tâches se rapproche plus des intérêts scolaires des étudiants en sciences pures. Les résultats des étudiants en sciences humaines pourraient donc être inférieurs à ceux des étudiants en sciences pures étant donné leur manque d'intérêt pour un tel contenu. Certaines recherches (Ausubel et Ausubel, 1968; Enwienne, 1976; Lovell, 1974; Torkia-Lagacé, 1981) tendent à montrer que la pensée abstraite émerge plus tôt dans les sciences exactes que dans les sciences humaines et sociales parce que dans le premier cas les étudiants peuvent confronter expérimentalement leurs idées en manipulant des contenus physiques.

Dans tous les.cas, que l'on considère les épreuves opératoires comme un biais favorable ou défavorable, la mesure des compétences cognitives d'étudiants en sciences humaines réclame un choix d'épreuves qui leur rende justice. Compte tenu du nombre restreint de recherches menées auprès d'étudiants universitaires et de la variabilité des résultats obtenus, on peut se demander si des étudiants en sciences humaines appliquées utilisent les schèmes formels théoriquement attendus pour résoudre des tâches opératoires de nature différente. La présente recherche revêt un caractère exploratoire et vise à apporter un éclairage supplémentaire à la compréhension du fonctionnement cognitif d'étudiants universitaires en sciences humaines appliquées. Dans cette perspective, quatre épreuves ont été retenues: La Quantification des probabilités, les Arrangements de lettres, le Contrôle des variables-billes, le Contrôle des variables-plantes. Les épreuves de 
Tableau no 1

Compte rendu synoptique des recherches evaluant

le niveau operatoire d'etudiants universitaires

\begin{tabular}{|c|c|c|c|c|c|c|c|c|c|c|c|}
\hline \multirow{2}{*}{$\begin{array}{l}\text { AUTEURS } \\
\text { (annee) }\end{array}$} & \multicolumn{4}{|c|}{ CARACTERISTIQUES DES SUJETS } & \multirow{2}{*}{$\operatorname{SCHEME}(S) / E P R E U V E(S)^{a}$} & \multicolumn{5}{|c|}{ \% DE SS PAR NIVEAU OPERATOIRE ${ }^{b}$} & \multirow{2}{*}{$\begin{array}{l}\text { METHODOLOGIE } \\
\text { TYPES DE MESURE }\end{array}$} \\
\hline & $\mathrm{Nb} / \mathrm{Sexe}$ & Age & Scolarite & Mi lieu. & & o.c. & $P-F$ & III A & INT & III $\mathrm{B}^{\mathrm{C}}$ & \\
\hline ADI $(1978)$ & $\begin{array}{rl}69 & F \\
6 & H\end{array}$ & $18-22$ & $\begin{array}{l}\text { Université - lere annee } \\
\text { Enseignement pré-scol. }\end{array}$ & & Balance & 84 & & 16 & & 0 & $\begin{array}{l}\text { Test papier-crayon } \\
\text {. Echelle en stades }\end{array}$ \\
\hline ARLIN (1975) & $60 \mathrm{~F}$ & & $\begin{array}{l}\text { Université }-2 e \text { annee } \\
\text { Psychologie de l'edu- } \\
\text { cation }\end{array}$ & & $\begin{array}{l}\text { Combinaison des corps chimi- } \\
\text { ques; pendule; projection des } \\
\text { ombres }\end{array}$ & 48.4 & & & & 51.6 & $\begin{array}{l}\text { Methode clinique } \\
\text { Analyse en stades } \\
\text { (Formel vs non- } \\
\text { formel) } \\
\text { Score global pour } \\
\text { les trois taches }\end{array}$ \\
\hline DESAUTELS (1978) & 95 & $\bar{x}: 17$ & $\begin{array}{l}\text { CEGEP: Sciences de la } \\
\text { sante - lêre année }\end{array}$ & & $\begin{array}{l}\text { Pendule } \\
\text { Permutation } \\
\text { - Conservation du mouvement } \\
\text { sur un plan horizontal } \\
\text { Au global }\end{array}$ & $\begin{array}{c}14.3 \\
11.4 \\
6.7 \\
10\end{array}$ & & \begin{tabular}{|l|}
33 \\
29.6 \\
21.1 \\
80
\end{tabular} & $\begin{array}{l}46.5 \\
57.8\end{array}$ & $\begin{array}{l}52.7 \\
15.5 \\
14.4 \\
10\end{array}$ & $\begin{array}{l}\text { Methode clinique } \\
\text { semi-standardisée } \\
\text { Echel le en stades }\end{array}$ \\
\hline $\begin{array}{l}\text { DILLING } \\
\text { WHEATLEY et } \\
\text { MITCHELL (1976) }\end{array}$ & $\begin{aligned} 19 \mathrm{~F} \\
8 \mathrm{H}\end{aligned}$ & $18-25$ & $\begin{array}{l}\text { Université } \\
\text { Sciences de l'educa- } \\
\text { tion. }\end{array}$ & & $\begin{array}{l}\text { Conservation du volume; } \\
\text { contróle des variables; balance }\end{array}$ & 39.2 & 28.8 & & & 32 & \\
\hline ELKIND (1962) & $\begin{aligned} 153 \mathrm{~F} \\
87 \mathrm{H}\end{aligned}$ & $17-37$ & $\begin{array}{l}\text { Universite - lêre annee } \\
\text { psychologie }\end{array}$ & & Conservation du volume & & & & & $F-52-58$ & $\begin{array}{l}\text { Test papier-crayon } \\
\text {. Réuss i te-échec }\end{array}$ \\
\hline ENWIEME (1976) & $\begin{array}{ll}56 \mathrm{~F} \\
46 \mathrm{H}\end{array}$ & & $\begin{array}{l}\text { Universite } \\
8 \text { concentrations diffe- } \\
\text { rentes }\end{array}$ & & 5 epreuves & & & & & $46.07^{\star}$ & $\begin{array}{l}\text { Test papier-crayon } \\
\text { - Echelle en points } \\
(0-100)\end{array}$ \\
\hline JOYCE (1977) & 66 & $19-22$ & $\begin{array}{l}\text { Universite - majeur en } \\
\text { education, section } \\
\text { enseignement des } \\
\text { sciences }\end{array}$ & & $\begin{array}{l}\text { Syllogisme } \\
\text { Combinaison des corps chimi- } \\
\text { ques } \\
\text { Balance } \\
\text { - Proportion } \\
\text { Pendule } \\
\text { Au total (i.e. reussite } 5 / 5 \text { ) }\end{array}$ & 7.6 & 15.2 & 51.5 & & $\begin{array}{l}100 \star \\
95.4 \\
92.4 \\
74.1 \\
33 \\
25.7\end{array}$ & $\begin{array}{l}\text { Me thode clinique (?) } \\
\text {. Réussite-échec }\end{array}$ \\
\hline JURASCHEK (1974) & $\mid \begin{array}{l}131 \\
19 \\
11\end{array}$ & & $\begin{array}{l}\text { a) Université - Educa- } \\
\text { tion, section ele- } \\
\text { mentaire } \\
\text { b) Université - educa- } \\
\text { tion, section } \\
\text { mathematiques } \\
\text { c) "Honors calcul us } \\
\text { students" }\end{array}$ & & $\begin{array}{l}\text { Balance; probabilite; combi- } \\
\text { naison des corps chimiques }\end{array}$ & $\begin{array}{l}52 \\
5 \\
0\end{array}$ & & & & $\begin{array}{l}5 \\
47 \\
64\end{array}$ & $\begin{array}{l}\text { Methode clinique } \\
\text { Anaiyse en stades } \\
\text { et en points }\end{array}$ \\
\hline
\end{tabular}


Tableau no 1 (suite)

\begin{tabular}{|c|c|c|c|c|c|c|c|c|c|}
\hline $\begin{array}{l}\text { KARPLUS et } \\
\text { KARPLUS }(1970)\end{array}$ & 83 & & $\begin{array}{l}\text { a) Professeurs de } \\
\text { sciences partici- } \\
\text { pant a un symposium } \\
\text { a propos de la théo- } \\
\text { rie piagetienne } \\
\text { b) professeurs de } \\
\text { physique }\end{array}$ & $\begin{array}{l}\text { Logique propositionnelle } \\
\text { (Islands Puzzle) }\end{array}$ & 86 & & 27 & 6 & - Test papier-crayon \\
\hline $\begin{array}{l}\text { KARPLUS, ADI, } \\
\text { LAWSON }(1980)\end{array}$ & $\begin{array}{l}33 \mathrm{~F} \\
53 \mathrm{H}\end{array}$ & & $\begin{array}{l}\text { Universjté - lère et } \\
\text { 2e annees (physique) }\end{array}$ & $\begin{array}{l}\text { Proportion } \\
\text { Probabilite } \\
\text { Correlation }\end{array}$ & $\begin{array}{r}13 \\
-2 \\
-2 \\
16\end{array}$ & $\begin{array}{r}6 \\
93 \\
16 \\
20\end{array}$ & & $\begin{array}{l}74 \\
79 \\
50\end{array}$ & $\begin{array}{l}\text { - Test papier-crayon } \\
\text {. Echelle en stades }\end{array}$ \\
\hline $\begin{array}{l}\text { LAWSON et RENNER } \\
(1974)\end{array}$ & 143 & $\begin{array}{l}\text { Med: } 18.6 \\
(16 ; 6-23)\end{array}$ & Universite - lère annese & $\begin{array}{l}\text { Conservation du volume (plas- } \\
\text { ticine); logique proposition- } \\
\text { nelle (implication reciproque); } \\
\text { pendule; flexibilité }\end{array}$ & 51 & 27 & & 22 & $\begin{array}{l}\text { Methode clinique } \\
\text {. Analyse en stades } \\
\text { et en points }\end{array}$ \\
\hline $\begin{array}{l}\text { LAWSON, KARPLUS, } \\
\text { ADI }(1978)\end{array}$ & $\begin{array}{l}35 \mathrm{~F} \\
52 \mathrm{H}\end{array}$ & $\bar{x}: 19,58$ & Université - lère annee & $\begin{array}{l}\text {. Proportion } \\
\text { Probabilite< } \\
\text {. Correlation } \\
\text {. Logique propositionnelle< }\end{array}$ & & & & $\begin{array}{l}80.4^{\star} \\
73.3 \\
71.6 \\
67.8 \\
67.1 \\
16.9 \\
16.2\end{array}$ & $\begin{array}{l}\text { - Test papier-crayon } \\
\text { - Réussite-echec }\end{array}$ \\
\hline $\begin{array}{l}\text { LAWSON, NORDLAND } \\
\text { et DeVITO }(1974)\end{array}$ & $\begin{aligned} 34 \mathrm{~F} \\
2 \mathrm{H}\end{aligned}$ & $\begin{array}{l}\text { Med: } 18.5 \\
(17.5- \\
20.2)\end{array}$ & $\begin{array}{l}\text { Universite - lere et } \\
2 e \text { annees (Sciences de } \\
\text { l'education - ler } \\
\text { cycle) }\end{array}$ & $\begin{array}{l}\text { Conservation du volume (plas- } \\
\text { ticine et cylindre): pendule; } \\
\text { flexibilité; balance }\end{array}$ & 39 & & 50 & 11 & $\begin{array}{l}\text { Méthode clinique } \\
\text { - Analyse en stades } \\
\text { et en points }(0-4)\end{array}$ \\
\hline $\begin{array}{l}\text { LAWSON, NORDLAND } \\
\text { et DeVITO }(1975)\end{array}$ & $\begin{aligned} 68 \mathrm{~F} \\
3 \mathrm{H}\end{aligned}$ & $\begin{array}{l}X: 18.6 \\
(18-20)\end{array}$ & $\begin{array}{l}\text { Université - lère et } \\
\text { 2e annees (Sciences de } \\
\text { i'education - ler } \\
\text { cycle) }\end{array}$ & $\begin{array}{l}\text { Conservation du volume } \\
\text { (cylindre) } \\
\text {. Flexibilite } \\
\text {. Pendule }\end{array}$ & $\begin{array}{l}46 \\
38 \\
31\end{array}$ & & $\begin{array}{l}54 \\
42 \\
55\end{array}$ & $\begin{array}{r}0 \\
20 \\
14\end{array}$ & $\begin{array}{l}\text { - Me thode clinique } \\
\text {. Ana lyse en stades } \\
\text { et en points }(0-4)\end{array}$ \\
\hline $\begin{array}{l}\text { MARSH et LOSEKE } \\
(1978)\end{array}$ & $\begin{array}{l}51 \\
22\end{array}$ & & $\begin{array}{l}\text { Universitế - lère annee } \\
\text { Psychologie } \\
\text { Chimie }\end{array}$ & $\begin{array}{l}\text { Angles d'incidence et de } \\
\text { reflexion; vases communi- } \\
\text { cants; balance; projection } \\
\text { des ombres }\end{array}$ & $\begin{array}{l}61 \\
77\end{array}$ & & $\begin{array}{l}35 \\
23\end{array}$ & $\begin{array}{l}4 \\
0\end{array}$ & - Test papier-crayon \\
\hline $\begin{array}{l}\text { MCKINNON et } \\
\text { RENNER (1971) }\end{array}$ & $\begin{array}{l}78 \mathrm{~F} \\
53 \mathrm{H}\end{array}$ & & Université - lère annêe & $\begin{array}{l}\text { Conservation du volume; } \\
\text { logique propositionnelie } \\
\text { (implication reciproque); } \\
\text { pendule; flexibilite }\end{array}$ & 50 & & 25 & 10 & $\begin{array}{l}\text { Methode clinique } \\
\text {. Analyse en stades } \\
\text { et en points }\end{array}$ \\
\hline
\end{tabular}




\begin{tabular}{|c|c|c|c|c|c|c|c|c|c|c|}
\hline MCKINNON (1978) & 185 & & $\begin{array}{l}7 \text { collëges et universi- } \\
\text { tés }\end{array}$ & divers & $\begin{array}{l}\text { - Conservation du valume } \\
\text { - Contróle des variables }\end{array}$ & $\begin{array}{l}28.1 \\
58.4\end{array}$ & & 13.5 & $\begin{array}{l}71.9^{*} \\
28.1\end{array}$ & Me thode clinique (?) \\
\hline PARETE (1979) & 231 & & Universite - lère annee & & $\begin{array}{l}\text { Pendule; triangle, flexibi- } \\
\text { lité; proportion }\end{array}$ & 50.8 & 10.4 & 29.1 & 10 & Test papier-crayon \\
\hline $\begin{array}{l}\text { PATTERSON et } \\
\text { MILAKOFSKY } \\
(1980)\end{array}$ & 210 & $18-19$ & $\begin{array}{l}\text { Université - lère et } \\
2 e \text { années }\end{array}$ & & $\begin{array}{l}\text { Conservation du volume. } \\
\text {. Egalité des angles d'inci- } \\
\text { dence } \\
\text { - Projection des ombres } \\
\text { - Probabilites }\end{array}$ & & & & $\begin{array}{l}77.5^{\star} \\
84.1^{\circ} \\
81.8 \\
82.3\end{array}$ & $\begin{array}{l}\text { Test papier-crayon } \\
\text { IPDT } \\
\text {. Echelle en points }\end{array}$ \\
\hline $\begin{array}{l}\text { PROTINSKY et } \\
\text { HUGSTON (1980) }\end{array}$ & $70 \mathrm{~F}$ & $\begin{array}{l}\bar{x}: 19.7 \\
(18-23)\end{array}$ & $\begin{array}{l}\text { Université - lêre année } \\
\text { Sciences humaines }\end{array}$ & & $\begin{array}{l}\text { Conservation du } \\
\text { volume Elkind } \\
\text { Lavatelli } \\
\text { Piaget }\end{array}$ & & & & $\begin{array}{l}91^{*} \\
63 \\
100\end{array}$ & Methode clinique \\
\hline ROSS (1973) & $\begin{array}{l}35 \mathrm{~F} \\
30 \mathrm{H}\end{array}$ & $x: 20.31$ & $\begin{array}{l}\text { Université - lẻre année } \\
\text { Education }\end{array}$ & & $\begin{array}{l}\text { Balance } \\
\text { Pendule } \\
\text { Correlation } \\
\text { Combinaison des corps chimi- } \\
\text { ques }\end{array}$ & $\begin{array}{r}32.4 \\
47.7 \\
1.5 \\
24.6\end{array}$ & & $\begin{array}{l}50.8 \\
21.5 \\
89.2 \\
70.8\end{array}$ & $\begin{array}{r}16.9 \\
30.8 \\
9.2 \\
4.6\end{array}$ & $\begin{array}{l}\text { Methode clinique } \\
\text { Analyse en stades }\end{array}$ \\
\hline SCHWEBEL (1975) & $\begin{array}{l}30 \mathrm{~F} \\
30 \mathrm{H}\end{array}$ & 19 & Université - lëre annęe & & $\begin{array}{l}\text { Balance; flexibilite; } \\
\text { plan incline }\end{array}$ & 17 & 63 & & 20 & $\begin{array}{l}\text { Methode clinique } \\
\text { Analyse en stades } \\
\text { et cotation en } \\
\text { points }(0-4)\end{array}$ \\
\hline $\begin{array}{l}\text { SILLS \& DUDLEY } \\
\text { HERON }(1976)\end{array}$ & $\begin{array}{l}26 \mathrm{~F} \\
29 \mathrm{H}\end{array}$ & & $\begin{array}{l}\text { Université - lëre année } \\
\text { Chimie }\end{array}$ & & $\begin{array}{l}\text { Combinaison des corps chimi- } \\
\text { ques }\end{array}$ & $\begin{array}{l}22.2 \\
17.8\end{array}$ & $\begin{array}{l}33.3 \\
35.7\end{array}$ & & $\begin{array}{l}44.5 \\
46.5\end{array}$ & $\begin{array}{l}\text { Test papier-crayon } \\
\text {. Me thode clinique }\end{array}$ \\
\hline $\begin{array}{l}\text { STROHM KITCHENER } \\
\text { et KING }(1981)\end{array}$ & $\begin{array}{l}20 \\
20\end{array}$ & $\begin{array}{l}19.6 \\
(19-20) \\
28.2 \\
(24-34)\end{array}$ & $\begin{array}{l}\text { Université - lère année } \\
\text { (arts) } \\
\text { Universite̊ - doctorat } \\
\text { (arts) }\end{array}$ & & $\begin{array}{l}\text { Combinaison des corps chimi- } \\
\text { ques; pendule }\end{array}$ & \multicolumn{4}{|c|}{ 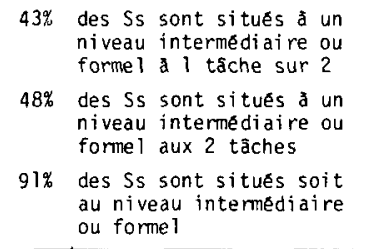 } & $\begin{array}{l}\text { Methode clinique } \\
\text { - Echelle en points } \\
(0-4)\end{array}$ \\
\hline $\begin{array}{l}\text { TOMLINSON- } \\
\text { KEASEY (1972) }\end{array}$ & $\begin{array}{ll}24 F \\
24 F\end{array}$ & $\begin{array}{l}\bar{X}: 19.7 \\
\bar{x}: 54\end{array}$ & Universitê - lère année & & Pendule; balance; flexibilite & $\begin{array}{l}44 \\
37\end{array}$ & & & $\begin{array}{l}23 \\
17\end{array}$ & $\begin{array}{l}\text { Methode clinique } \\
\text { Echelle en stades }\end{array}$ \\
\hline
\end{tabular}




\begin{tabular}{|c|c|c|c|c|c|c|c|c|c|}
\hline $\begin{array}{l}\text { TORKIA-LAGACE } \\
(1981)\end{array}$ & \begin{tabular}{|l}
$3003 \mathrm{~F}$ \\
$2875 \mathrm{H}$
\end{tabular} & $\begin{array}{l}16-22 \\
85 \% \text { de } \\
1 \text { 'echan. } \\
\text { est âge } \\
\text { de } 17-18\end{array}$ & $\begin{array}{l}\text { College 1 } \\
\text { (20 concentrations } \\
\text { differentes) }\end{array}$ & $\begin{array}{l}\text { - Combinatoire } \\
\text {. Proportion } \\
\text { - Pourcentage ( } \\
\text { - Enigmes (rais } \\
\text { hypothet i co-d }\end{array}$ & & $\begin{array}{l}33.2 \\
30.0 \\
35.7 \\
63.2\end{array}$ & $\begin{array}{l}46.3 \\
27.6 \\
20.0\end{array}$ & $\begin{array}{l}20.5 \\
42.5 \\
64.3 \\
16.8\end{array}$ & $\mid \begin{array}{l}\text { - Test papier-crayon } \\
\text { - Echelle en stades }\end{array}$ \\
\hline $\begin{array}{l}\text { TORKIA-LAGACE } \\
(1981) \\
\text { (suite) }\end{array}$ & $\begin{array}{r}\text { a) } 167 \mathrm{~F} \\
633 \mathrm{H} \\
\text { b) } 288 \mathrm{~F} \\
346 \mathrm{H} \\
\text { c) } 166 \mathrm{~F} \\
32 \mathrm{H}\end{array}$ & & $\begin{array}{l}\text { a) Sciences pures et } \\
\text { appliqués } \\
\text { b) Sciences humaines } \\
\text { sans mathematiques } \\
\text { c) Techniques d'educa- } \\
\text { tion spécialisee, } \\
\text { assistance sociale, } \\
\text { technique de docu- } \\
\text { mentation }\end{array}$ & $\begin{array}{l}\text { Combinatoire } \\
\text {. Proportion } \\
\text {. Enigmes }\end{array}$ & $\begin{array}{l}\text { a) } \\
\text { b) } \\
\text { c) } \\
\text { a) } \\
\text { b) } \\
\text { c) } \\
\text { a) } \\
\text { b) } \\
\text { c) }\end{array}$ & $\begin{array}{l}14.1 \\
49.6 \\
42.9 \\
10.5 \\
43.8 \\
43.9 \\
30.8 \\
81.8 \\
74.0\end{array}$ & \begin{tabular}{|l}
45.2 \\
39.2 \\
51.3 \\
17.3 \\
32.6 \\
26.8 \\
27.2 \\
10.0 \\
20.8 \\
\end{tabular} & $\begin{array}{r}40.7 \\
11.2 \\
5.8 \\
72.2 \\
23.6 \\
29.3 \\
42.0 \\
8.2 \\
5.2 \\
\end{array}$ & \\
\hline $\begin{array}{l}\text { WALKER, HENDRIX } \\
\text { et MERTENS } \\
\text { (1979) }\end{array}$ & 86 & $18-27$ & $\begin{array}{l}\text { Université - lère année } \\
\text { (génétique) }\end{array}$ & $\begin{array}{l}\text { Logique proposi } \\
\text { combinatoire: } \\
\text { hypothetico-ded } \\
\text { (syllogismes) }\end{array}$ & & $28(\mathrm{no}$ & me1) & $72^{\star}$ & - Test papier-crayon \\
\hline WASSON (1969) & $\begin{array}{l}14 \mathrm{~F} \\
18 \mathrm{H}\end{array}$ & & $\begin{array}{l}\text { Universite - ler cycle } \\
\text { et 3e cycle }\end{array}$ & Logique proposi & & & & 6.2 & \\
\hline $\begin{array}{l}\text { WILLIAM et al. } \\
(1978)\end{array}$ & $\begin{array}{l}40 \mathrm{~F} \\
21 \mathrm{H}\end{array}$ & $\begin{array}{l}19.11 \\
(17.9- \\
29.9)\end{array}$ & Université - ler cycle & $\begin{array}{l}\text {. Logical reaso } \\
\text { (Burney, } 1974 \\
\text { Combinatoire } \\
\text { Analog) }\end{array}$ & ectric & $\begin{array}{l}\text { Le res } \\
\text { aux au } \\
\text { situen }\end{array}$ & $\begin{array}{l}\text { de } \\
\text { que } \\
\text { nivea }\end{array}$ & $\begin{array}{l}65.57^{*} \\
\text { dire } \\
\text { A }\end{array}$ & $\begin{array}{l}\text { - Test papier-crayon } \\
\text {. Methode clinique } \\
\text { (cotation en } \\
\text { points } 0-10 \text { ) }\end{array}$ \\
\hline
\end{tabular}

a Le point (.) précếdant dans certains cas (ex.: Joyce, 1977) les épreuves utilisées signifie que les rêsultats sont présentês épreuves par êpreuves; l'absence du point signifie que les ressultats sont presentés globalement.

b Les niveaux opératoires retenus sont les suivants: opératoire concret (O.C.); pré-formel ( $P-F)$; seuil de l'opératoire formel (IIIA); niveau intermédiaire entre IIIA et IIIB (INT), et phase d'achévement du niveau opêratoire formel (IIIB).

${ }^{c}$ L'asterique $\left(^{\star}\right)$ joint aux résultats de certaines recherches signifie que le type de mesure utilisé ne permet pas d'échelonner les résultats sur le. continuum concret-formel. 
Quantification des probabilités et des Arrangements de lettre sont des épreuves dont les problèmes peuvent être résolus à l'aide des connaissances scolaires acquises, sans une compréhension formelle de la notion. Les deux épreuves du Contrôle des variables (billes et plantes) font référence à un schème non appris de façon spécifique à l'école, le Contrôle des variables-billes impliquant un contenu physique, le Contrôle des variables-plantes un contenu quotidien et familier.

\section{MÉTHODOLOGIE}

\section{Sujets}

Les sujets de cette étude $(n=48)$ sont tous étudiants à l'université en dernière année du baccalauréat en sciences humaines appliquées. Le groupe 1 comprend 20 étudiants ( 16 femmes, 4 hommes) dont l'âge varie entre 20 ans, 10 mois et 24 ans $(\bar{X}=22.7$ E.T. $=10$ mois). Le groupe 2 comprend 28 étudiants $(21$ femmes, 7 hommes) dont l'âge varie entre 20 ans, 10 mois et 43 ans $(\bar{X}=24.9$ E.T. $=5.4$ mois). Trois sujets seulement sont âgés de plus de 30 ans, ce qui confère une certaine homogénéité aux groupes. La proportion des sujets masculins et féminins reflète bien la clientèle inscrite à ce programme.

\section{Procédure}

Les épreuves papier-crayon ont été présentées en classe en passation collective à chaque groupe lors d'une session d'une durée de 90 minutes. Le groupe 1 a complété les épreuves du Contrôle des variables-billes et de la Quantification des probabilités. Le groupe 2 a répondu aux questionnaires des Arrangements de lettres et du Contrôle des variables-plantes. Compte tenu des contingences horaires, le temps n'a pas permis la passation des quatre épreuves par les 48 étudiants.

\section{Instruments}

\section{Quantification des probabilitès}

Nous inspirant d'un certain nombre de travaux (Dupont, Gendre et Pauli, 1975; Higelé, 1978; Larivée, 1977; Laroche, 1966; Longeot, 1969; Longeot et al., 1974; Nassefat, 1963; Piaget et Inhelder, 1951; Schircks et Laroche, 1970) nous avons constitué un test papier-crayon de Quantification des probabilités à passation collective.

On présente à l'étudiant deux sacs contenant un certain nombre de boules blanches et de boules noires. Celui-ci doit répondre à la question suivante: "Si tu veux tirer une seule boule noire, dans lequel des deux sacs a-t-on le plus de chances d'en tirer une du premier coup?" Trois choix de réponses sont possibles: "dans le sac A", "dans le sac B", "les chances sont égales". L'étudiant doit justifier sa réponse.

La composition, la nature opératoire et la correction des dix items (voir tableau 2) ont été établies par Piaget et Inhelder (1951) et vérifiẻes ultérieurement (Nassefat, 1963; Longeot, 1966, 1969; Longeot et al., 1974). 
Tableau no 2

Classement des items de l'épreuve de Quantification des probabilites seion leur niveau operatoire

\begin{tabular}{|c|c|c|c|c|}
\hline \multirow{2}{*}{ ITEMS } & \multicolumn{4}{|c|}{ Niveaux operatoires } \\
\hline & Operatoire & Intermediaire & Formel A & Forme $1 \mathrm{~B}$ \\
\hline & $\begin{array}{l}(2)^{a} 2 / 4-3 / 7 \\
(7)^{3 / 8}-2 / 8\end{array}$ & $\begin{array}{l}\text { (1) } 1 / 2-2 / 4 \\
\text { (8) } 1 / 2-3 / 6\end{array}$ & $\begin{array}{l}\text { (9) } 4 / 6-2 / 3 \\
\text { (4) } 3 / 12-1 / 4 \\
(6) 6 / 9-4 / 6\end{array}$ & $\begin{array}{r}\text { (3) } 2 / 6-3 / 8 \\
(5) 1 / 3-2 / 5 \\
(10) 3 / 5-5 / 9\end{array}$ \\
\hline
\end{tabular}

a les chiffres entre parentheses indiquent l'ordre de presentation des items

Un item est réussi lorsque l'étudiant fournit la bonne réponse et donne une justification adéquate. Une justification est considérée adéquate quand l'étudiant prend en considération les trois informations pertinentes (les cas favorables, défavorables et possibles) et les compose en établissant un rapport proportionnel ou en utilisant la notion de fraction ("C'est égal, parce que 3/12 simplifié, c'est $1 / 4$, et ce qui compte, ce sont les proportions"). Une justification est considérée inadéquate lorsque l'étudiant ne prend en considération qu'une partie des informations pertinentes ("En A (1/3) parce qu'il y a moins de boules que dans l'autre sac (2/5)'').

Quatre niveaux opératoires sont définis: opératoire concret (réussite à 0,1 ou 2 items concrets et échec aux autres items), intermédiaire (réussite aux items concrets et à au moins un des deux items intermédiaires et échec aux autres items), formel $\mathrm{A}$ (réussite à au moins deux items formels $\mathrm{A}$ et réussite aux items des niveaux précédents et échec aux items formels B), formel B (réussite à au moins deux items formels et à tous les items des niveaux précédents).

\section{Arrangement de lettres}

Le test papier-crayon à passation collective et la grille de correction élaborés pour l'épreuve des arrangements s'inspirent principalement des travaux de Laveault (1981), Longeot (1978) et Noëlting et al. (1980). L'étudiant doit faire soit tous les duos, soit tous les trios, soit tous les quatuors de lettres tirés d'un ensemble de 2, 3 ou 4 lettres, après avoir anticipé combien il peut en faire dans chacun des cas. Le test comprend 12 items dont six requièrent une exécution effective et six une anticipation du nombre d'arrangements possibles ou l'énonciation de la règle du carré pour les arrangements de deux lettres dans " $n$ " et une généralisation de la règle des arrangements de " $y$ " lettres dans " $x$ ". Leur composition et leur nature opératoire sont présentées au tableau 3.

La réussite à un item d'exécution dépend non seulement du nombre d'arrangements trouvés mais aussi de la stratégie utilisée par l'étudiant dans la réalisation des arrangements. Les items d'exécution de niveau concret sont réussis lorsque l'ensemble des arrangements est présenté selon, soit l'emploi du produit cartésien (Longeot, 1978), soit la stratégie dite "fixation-variation"; cette dernière consiste à "fixer" une lettre et à faire "varier" l'autre selon toutes les possibilités, puis à 
Tableau no 3

Classement des items de l'epreuve des Arrangements

de lettres selon leur niveau operatoire

\begin{tabular}{|c|c|c|c|c|c|c|c|}
\hline \multirow[t]{2}{*}{ ITEMS } & \multicolumn{7}{|c|}{ NIVEAUX OPERATOIRES } \\
\hline & Concret & & Formel Al & & Forme 1 A2 & & Forme $1 \mathrm{~B}$ \\
\hline & $\begin{array}{l}\text { (1) }{ }^{a} \text { realisation } \\
(p=2 ; n=2)\end{array}$ & (4) & $\begin{array}{l}\text { anticipation } \\
(p=2 ; n=5)\end{array}$ & (8) & $\begin{array}{l}\text { realisation } \\
(p=3 ; n=3)\end{array}$ & (9) & $\begin{array}{l}\text { anticipation } \\
(p=3 ; n=4)\end{array}$ \\
\hline & $\begin{array}{l}\text { (2) realisation } \\
(p=2 ; n=3)\end{array}$ & (5) & $\begin{array}{l}10 i \text { du carré } \\
(\mathrm{p}=2)\end{array}$ & $(10)$ & $\begin{array}{l}\text { realisation } \\
(p=4 ; n=2)\end{array}$ & (11) & $\begin{array}{l}\text { anticipation } \\
(p=4 ; n=3)\end{array}$ \\
\hline & $\begin{array}{l}\text { (3) réalisation } \\
(p=2 ; n=4)\end{array}$ & (6) & $\begin{array}{l}\text { anticipation } \\
(p=2 ; n=9)\end{array}$ & & & (12) & $\begin{array}{l}\text { généralisation } \\
\text { de la loi } \\
(p=x ; n=y)\end{array}$ \\
\hline & & (7) & $\begin{array}{l}\text { realisation } \\
(p=3 ; n=2)\end{array}$ & & & & \\
\hline
\end{tabular}

a les chiffres entre parentheses indiquent l'ordre de presentation des items

passer à une autre lettre et à exploiter à nouveau les variations possibles de la seconde lettre (Cloutier, 1982; Larivée et Normandeau, 1984; Noëlting et al., 1980). Les items d'exécution de niveau formel sont réussis lorsque l'ensemble des arrangements est présenté selon une stratégie de "fixation-variation hiérarchisée". Il s'agit en fait d'une généralisation de la même stratégie de niveau concret.

Les items d'anticipation et d'énoncé de loi, sont tous de niveau formel puisque l'étudiant doit dégager des principes à partir de l'observation du réel, dépasser la situation concrète pour en tirer finalement des lois généralisables i.e. applicables à tous les cas possibles (Cloutier 1982).

Quatre niveaux différents sont distingués: opératoire concret (réussite à 1,2 ou 3 items concret et échec aux items suivants), formel Al (réussite aux items concrets et à au moins 3 des 4 items formels $\mathrm{A} 1$ et échec aux autres items), formel A 2 (réussite aux items concrets et formels A1 et à au moins 1 des 2 items formels A2 et échec aux autres items), formel B (réussite aux items concrets, formels A 1, formels A 2 et à au moins 2 des 3 items formels B).

\section{Contrôle des variables}

Billes:

Cette épreuve s'inspire des travaux de Karplus et al. $(1975,1977)$ et de Wollman (1977 a, b). Quatre expériences dont le contenu est lié au domaine de la physique sont présentées à l'étudiant. Le dispositif présenté graphiquement comprend une rampe de lancement et des billes dont le poids et la composition varient. L'étudiant doit réfléchir à propos de la force d'impact d'une bille en mouvement partant à des hauteurs diverses lorsqu'elle heurte une bille immobile, tout en considérant les facteurs impliqués (hauteur de chute de la bille de lancement, nature et poids de la bille de lancement et de la bille-cible). 
Plantes:

Cette tâche extraite de la recherche de Kuhn et Brannock (1977) présente un contenu de la vie quotidienne: un ensemble de plantes d'intérieur auxquelles sont donnés des soins hebodomadaires différents (petite ou grande quantité d'eau, engrais à faible ou haute teneur en potasse, lotion pour les feuilles). Deux d'entre elles sont en santé, les deux autres ne sont pas en santé. L'étudiant doit alors démontrer, hors de tout doute, lequel (lesquels) parmi les facteurs identifiés est (sont) essentiel(s) pour la santé de ces plantes.

Le schéma d'analyse élaboré pour les épreuves du contrôle des variables s'inspire des recherches Karplus et al. (1975, 1977), Kuhn et Brannock (1977), Inhelder et Piaget (1955), Somerville (1974) et Wollman (1977 a, b). Quatre niveaux opératoires (concret, intermédiaire, formel $\mathrm{A}$, formel $\mathrm{B}$ ) sont définis en fonction des rêponses et des justifications fournies par l'étudiant. Par exemple, un étudiant de niveau opératoire concret peut faire varier plus d'un facteur à la fois, se centrer sur un facteur en oubliant les interactions entre les autres, avoir des raisonnements basés sur des intuitions et des connaissances personnelles. Un étudiant de niveau intermédiaire peut être incapable de concevoir qu'un facteur soit inopérant, isoler le facteur opérant sans pouvoir exclure les autres facteurs, avoir de la difficulté à dissocier l'effet respectif de chacun des facteurs. Un étudiant de niveau formel A utilisant des raisonnements relativement systématiques peut soit exclure logiquement un facteur non-opérant sans toutefois être capable d'isoler le facteur opérant, soit isoler le facteur opérant tout en excluant partiellement les facteurs non-opérants. Un étudiant de niveau formel B utilise des raisonnements systématiques et applique de façon adéquate le scheme "toutes choses étant égales d'ailleurs". Il peut isoler le facteur opérant et exclure logiquement des facteurs non-opérants, identifier l'effet propre à chacun des facteurs.

\section{RÉSULTATS}

Les tableaux 4 et 5 présentent la fréquence des sujets aux différents niveaux opératoires pour chacune des épreuves. Les résultats mettent en évidence des pourcentages variables de sujets ayant atteint soit le niveau formel B soit le niveau formel A ou. B.

Tableau no 4

$$
\begin{aligned}
& \text { Fréquence }(\%) \text { des sujets du groupe } 1 \text { aux différents niveaux } \\
& \text { opēratoires à l 'épreuve du contrôle des variables-billes } \\
& \text { et à l'épreuve des probabilités }
\end{aligned}
$$

\begin{tabular}{lcccc}
\hline \multirow{2}{*}{ Epreuves } & \multicolumn{4}{c}{ Niveaux opératoires } \\
\cline { 2 - 5 } & Concret & Intermediaire & Formel A & Formel B \\
\hline $\begin{array}{l}\text { Controle des } \\
\text { variables } \\
\text { (billes) }\end{array}$ & $10(50)$ & $3(15)$ & $3(15)$ & $4(20)$ \\
\begin{tabular}{l} 
Probabilite \\
\hline
\end{tabular} & - & $2(10)$ & $4(20)$ & $14(70)$ \\
\hline
\end{tabular}


Tableau no 5

Frequence (\%) des sujets du groupe 2 aux differents niveaux opēratoires à l'épreuve du contrôle des variables-plantes et à l'épreuve des arrangements

\begin{tabular}{lccccc}
\hline \multirow{2}{*}{ Epreuves } & \multicolumn{5}{c}{ Niveaux operatoires } \\
\cline { 2 - 6 } & Concret & Intermediaire & Formel A1 & Formel A2 & Formel B \\
\hline $\begin{array}{l}\text { Controle des } \\
\text { variables } \\
\text { (plantes) }\end{array}$ & $6(21.4)$ & $8(28.6)$ & - & $3(10.7)$ & $11(39.3)$ \\
Arrangements & $2(7.1)$ & - & $9(32.2)$ & $7(25)$ & $10(35.7)$ \\
\hline
\end{tabular}

Des comparaisons intragroupes évaluées à l'aide du test McNemar pour échantillons reliés permettront de vérifier l'existence d'une différence quant au niveau opératoire atteint entre une tâche à contenu scolaire et une tâche à contenu non scolaire. Il existe une différence significative quant à la distribution des étudiants du groupe 1 entre l'épreuve du Contrôle des variables-billes et l'épreuve de la Quantification des probabilités $\left(\mathrm{X}^{2}=9.09, \mathrm{dl}=1, \mathrm{p}<.01\right)$ ainsi qu'entre les épreuves du Contrôle des variables-plantes et l'épreuve des Arrangements de lettres $\left(\mathrm{X}^{2}=7.56, \mathrm{dl}=1, \mathrm{p}<.01\right)$ pour les étudiants du groupe 2. Dans le premier cas, les étudiants sont majoritairement de niveaux non-formels à l'épreuve du Contrôle des variables-billes et presque tous aux niveaux formels à l'épreuve de la Quantification des probabilités. Dans le second cas, on observe une distribution égale entre les niveaux non-formels et formels à l'épreuve du Contrôle des variablesplantes alors que presque tous les étudiants sont de niveaux formels à l'épreuve des Arrangements de lettres.

Des comparaisons intergroupes mesurées à 1'aide d'un Chi-carré permettront de vérifier l'impact du contenu sur la réussite à une tâche du Contrôle des variables et la similarité ou la différence du niveau opératoire atteint entre deux tâches à contenu scolaire. Les comparaisons intergroupes s'avèrent non significatives entre l'épreuve du Contrôle des variables-plantes et celle du Contrôle des variablesbilles $\left(\mathrm{X}^{2}=.54, \mathrm{dl}=1, \mathrm{p}<.25\right)$ de même qu'entre les épreuves de Quantification des probabilités et d'Arrangements de lettres $\left(\mathrm{X}^{2}=.03, \mathrm{dl}=1, \mathrm{p}<.45\right)$.

Les comparaisons intragroupes permettent de mettre en évidence des niveaux de réussite différents selon que l'épreuve relève d'un acquis scolaire ou non. Les comparaisons intergroupes infirment l'existence d'un effet du contenu sur le niveau de réussite aux épreuves du Contrôle des variables. De même le niveau de réussite opératoire entre les deux épreuves à contenu scolaire ne diffère pas.

\section{CONCLUSIONS}

Les résultats de la présente recherche sont comparables à ceux obtenus dans les études antérieures. Le pourcentage de réussite au niveau formel A ou B aux épreuves du Contrôle des variables est nettement inférieur à celui des épreuves de Probabilité ou de Combinatoire (Desautels, 1978; Joyce, 1977; Ross, 1973). 
Malgré cette concordance globale avec les recherches antérieures, certains aspects des présents résultats soulèvent des questions. Comment expliquer par exemple le pourcentage de réussite au niveau formel A ou B relativement élevé aux épreuves d'arrangements et de probabilité et le pourcentage relativement faible aux deux épreuves du contrôle des variables; comment expliquer que des étudiants à la fin de leur baccalauréat aient pu réussir des études universitaires sans posséder les instruments nëcessaires?

Les résultats obtenus laissent croire que le schème du contrôle des variables est simplement plus tardivement acquis que celui de la combinatoire ou des probabilités. Toutefois deux éléments contredisent cette apparence. Premièrement, le schème du contrôle des variables est considéré comme l'un des premiers acquis formels (Larivée et Cormier, 1982, 1983; Neimark, 1975). Deuxièmement, la stratégie de fixation-variation requise pour réussir l'épreuve des Arrangements s'apparente à l'application du principe du contrôle des variables puisqu'il s'agit par exemple, de garder constante une lettre et de faire varier les autres. Ce dernier constat suggère que l'apprentissage scolaire pourrait expliquer les résultats élevés aux épreuves de l'Arrangement des lettres et de la Quantification des probabilités.

Une autre interprétation concerne la dichotomie compétence-performance. Les mesures recueillies constituent la performance de l'étudiant mais ne reflètent que plus ou moins fidèlement sa compétence. L'atteinte d'un niveau opératoire plus élevé à la tâche des Arrangements de lettres n'est peut-être pas liée principalement à la compréhension réelle du principe du contrôle des variables mais plutôt à un apprentissage scolaire. Une lecture attentive des protocoles individuels pour cette tâche permet de remarquer qu'un bon nombre d'étudiants offrent des réponses correctes aux items d'anticipation, formulent adéquatement les règles de généralisation. Cependant ils ne proposent aucune justification à l'appui. Le test papier-crayon ne permet pas de distinguer si de telles réponses sont attribuables au vernis scolaire (performance) ou à une réelle compréhension (compétence). D'autres étudiants encore, réussissent les items d'anticipation mais sont incapables de faire la liste exhaustive des arrangements de niveau formel A2.

L'absence d'une différence entre les deux épreuves du Contrôle des variables ne permet pas quant à elle d'évoquer l'influence du contenu sur la réussite opératoire comme nombre d'auteurs le signalent (Kuhn et Brannock, 1977; HigginsTrenk et Gaite, 1971; Horneman, 1974; Laveault, 1981; Leplat, Pailhous et Vermersch, 1975; Lunzer, Harrison et Davey, 1972; Sinnot, 1975).

Si les tâches du contrôle des variables telles que présentées aux étudiants sont valides, si elles mesurent bien l'utilisation que fait un étudiant du principe du contrôle des variables, si ce schème est une habileté cognitive que les adultes devraient posséder et pouvoir utiliser aisément, alors on peut s'interroger sur la non-acquisition de ce schème par les étudiants compte tenu de leur réussite scolaire. Ces données semblent en désaccord avec bon nombre de recherches (Cantu et Herron, 1978; Desautels, 1978; Karplus et al., 1977; Lawson et Renner, 1975; Lawson et al., 1975; Sheehan, 1970) montrant la présence d'une corrélation entre le niveau opératoire et la réussite scolaire. 
D'autre part, si les résultats sont justes on est alors en droit de se demander si les méthodes d'enseignement proposées et les modalités d'évaluation font vraiment appel au maniement de l'abstraction et des habiletés formelles. Peut-être que d'autres composantes cognitives, telle la mémoire, suffisent pour rencontrer les exigences académiques de certains programmes universitaires. (Herron, 1978).

La présente recherche met en évidence encore une fois certaines lacunes quant aux schèmes formels des étudiants de niveau universitaire. Si on accepte l'hypothèse interactionniste de la théorie piagétienne quant au développement opératoire formel et les tâches utilisées pour le mesurer on doit alors tenter de cerner les causes; les variables susceptibles d'être responsables de tels résultats. L'acceptation du point de vue interactionniste de la théorie opératoire nous incite alors à questionner les méthodes d'enseignement et les méthodes d'évaluation comme facteurs susceptibles d'influencer le niveau de développement opératoire.

\section{RÉFÉRENCES}

Adi, H. (1978). Intellectual development and reversibility of thought in equation solving. Journal for Research in Mathematics Education, 9(2), 204-213.

Arlin, P.K. (1975). Cognitive development in adulthood: a fifth stage? Developmental Psychology, 11 (5), 602-606.

Ausubel, D.P. \& Ausubel, P. (1968). Cognitive development in adolescence. Review of Educational Research, 36(4), 403-413.

Barnes, G. (1977). Scores on a Piaget-type questionnaire versus semester grades for lowerdivision college physics students. American Journal of Physics, 45 (9), 841-847.

Cantu, L.L.. \& Herron, J.D. (1978). Concrete and formal piagetian stages and science concept attainment. Journal of Research in Science Teaching, 15(2), 135-143.

Cloutier, R. (1982). Psychologie de l'adolescence. Chicoutimi= Gaëtan Morin.

Desautels, P. (1978). La pensée formelle. Montréal: CEGEP de Rosemont, Département de physique, Projet PROSIP (D.G.E.C.).

Dilling, R.A., Wheatley, G.H.\& Mitchell, R. (1976). An EEG investigation of the differences in the hemispheric specialization of formal and concrete operational persons. Paper presented at the Annual Meeting of the American Educational Research Association (San Francisco; California, April 19-23, 1976). US Department of Health Education \& Welfare National Institute of Education. (Eric ed 131354 )

Dupont, J.-B., Gendre, F. \& Pauli, L. (1975). Epreuves opératoires et tests factoriels. Cahiers Vilfredo Pareto, Tome 13 (35), 137-198.

Elkind, D. (1962). Quantity conceptions in college students. The Journal of Social Psycho$\log y, 57,459-465$.

Enwieme, A.A.E. (1976). The incidence of formal operations of students in eight subject areas of the nustep program at the University of Nebraska, (Doctoral dissertation, University of Nebraska-Lincoln, 1976). Dissertation Abstracts International, 37A, 2761-2762.

Good, R.G. (1977). How Children Learn Science: Conceptual Development and Implications for Teaching. New York: MacMillan.

Herron, J.D. (1978). Piaget in classroom. Journal of Chemical Education, 55 (3), 165-1 70.

Higelé, P. (1978). Etude de la mesure du niveau opératoire des adultes. Psychologie Française, 23(2), 95-105. 
Higgins-Trenk, A. \& Gaite, A.J.H. (1971). Elusiveness of formal operations thought in adolescents. Proceedings of the 79 th Annual Convention of American Psychological Association, 6, 201-202.

Horneman, J. (1974). L'influence du contenu sur la résolution des problèmes logiques. Enfance, 12, 45-64.

Inhelder, B. \& Piaget, J. (1955). De la logique de l'enfant à la logique de l'adolescent. Paris: Presses Universitaires de France.

Joyce, L.K. (1977). A study of formal reasoning in elementary education majors. Science Education, 61(2), 153-158.

Juraschek, W.A. (1974). Piagetian Cognitive Development among Prospective Teachers. (Technical report no 4). Austin University, Mathematics Education Center, (Eric Document Reproduction Service, No Ed 106 122).

Karplus, E.F. \& Karplus, R. (1970). Intellectual development beyond elementary school I. Deductive Logic. School Science and Mathematics, 60(5), 398-406.

Karplus, R. et al. (1975). Proportional Reasoning and Control of Variables in Seven Countries. Berkeley: Lawrence Hall of Science.

Karplus, R. et al. (1977). A survey of proportional reasoning and control of variables in seven countries. Journal of Research in Science Teaching, 14(5), 411-417.

Karplus, R. et al. (1980). Intellectual development beyond elementary school VIII: Proportional, probabilistic and correlational reasoning. School Science and Mathematics, 80, 673-683.

Kuhn, D. \& Brannock, J. (1977). Development of the isolation of variables scheme in experimental and "natural experiment" contexts. Developmental Psychology, 13(1), 9-14.

Larivee, S. (1977). Le fonctionnement cognitif de l'adolescent délinquant. Thèse de doctorat inédite. Université de Lausanne, Lausanne.

Larivee, S. \& Cormier, P. (1982). Le schème du contrôle des variables, un instrument du raisonnement scientifique. 1. Sa nature. Spectre, 12(1), 12-16.

Larivee, S. \& Cormier, P. (1983). Le schème du contrôle des variables, un instrument du raisonnement scientifique. 2. Ses implications. Spectre, $12(2), 24-28 ; 34$.

Larivee, S. \& Normandeau, S. (1984). Mattrise du schème de la combinatoire (permutations) chez des adolescents en classes spéciales. Revue canadienne d'éducation, accepté.

Laroche, J.L. (1966), Diagnostic des structures opératoires d'adultes de niveau C.E.P. Nancy: Institut National pour la formation des adultes (INFA).

Laveault, D. (1981). Le passage des opérations concrètes aux opérations formelles: une étude des séquences du développement des notions d'arrangement et de proportion, considérées dans des univers de tâche et de contenu variés. Thèse de doctorat inédite, Université Laval, Québec.

Lawson, A.E. \& Renner, J.W. (1974). A quantitative analysis of responses to Piagetian tasks and its implications for curriculum. Science Education, 58 (4), 545-560.

Lawson, A.E. \& Renner, J.W. (1975). Relationships of science subject matter and developmental levels of learners. Journal of Research in Science Teaching, 12 (4), 347-358.

Lawson, A.E., Karplus, R. \& Adi, H. (1978). The acquisition of propositional logic and formal operational schemata during the secondary school years. Journal of Research in Science Teaching, 15(6), 465-478.

Lawson, A.E., Nordland, F.H. \& DeVito, A. (1974). Piagetian formal operational tasks: A crossover study of learning effect and reliability. Science Education, 58(2), 267-276.

Lawson, A.E., Nordland, F.H. \& DeVito, A. (1975). Relationship of formal reasoning to 
achievement, aptitudes, and attitudes in preservice teachers. Journal of Research in Science Teaching, 12(4), 423-431.

Leplat, J., Pailhous, J. \& Vermersch, P. (1975). L'acquisition d'un système de represéntation est-elle rationalisable? Bulletin de psychologie. 28(315), 398-409.

Longeot, F. (1966). Expérimentation d'une échelle individuelle du développement de la pensée logique. BINOP, 22(5), 306-319.

Longeot, F. (1969). Psychologie différentielle et théorie opératoire de l'intelligence. Coll. Sciences du comportement, Paris: Dunod.

Longeot, F. (1978). Les stades opératoires de Piaget et les facteurs de l'intelligence. Grenoble: Presses Universitaires de Grenoble.

Longeot, F., Girard, P., Lambert, J.L. \& Rouquier, C. (1974). Echelle de développement de la pensée logique. Manuel d'instructions. Issy-les-Moulineaux: Editions scientifiques et psycho-techniques.

Lovell, K. (1974). Intellectual aspects of adolescence. In M.K. Pringle \& V.P. Varnia (Eds.). Advances in Educational Psychology, 2 (pp. 208-216). London: University of London Press.

Lunzer, E.A., Harrison, C. \& Davey, M. (1972). The four-card problem and the generality of formal reasoning. Quarterly Journal of Experimental Psychology, 24, 326-339.

Marsh, G. \& Loseke, D. (1978). Formal operations in the college classroom. In G.J. Lubin, M.K. Poulsen, J.F. Magary, M. Soto-McAlister (Eds.). Piagetian Theory and its Implications for the Helping Professions. Proceedings Seventh Interdisciplinary Conference (vol. II) (pp. 314-317). Los Angeles, California: University of Southern California.

McKinnon, J.W. (1978). The college student and formal operations. In Renner et al.(Eds). Research, Teaching, and Learning with the Piaget Model. Norman: University of Oklahoma, p. 110-129.

McKinnon, J.W. \& Renner, J.W. (1971). Are colleges concerned with intellectual development? American Journal of Physics, 39, 1047-1052.

Nassefat, M. (1963). Etude quantitative sur l'évolution des opérations intellectuelles. Neuchatel: Delachaux et Niestlé.

Neimark, E.D. (1975). In tellectual development during adolescence. In F.D. Horowitz (Ed.). Review of Child Development Research (Vol. IV). Chicago: University of Chicago Press.

Noelting, G. et coll. (1980). Stadex: nombre, hasard et classe. Québec: Ecole de Psychologie, Université Laval.

Parete, J.D. (1979): Formal reasoning abilities of college age students: an investigation of the concrete and formal reasoning stages formulated by Jean Piaget. Dissertation Abstracts International, 39(10A), 6006.

Patterson; H.O. \& Milakofsky, L. (1.980). A paper-and-pencil inventory for the assessment of Piage t's tasks: Applied Psychological Measurement, 4(3), 341-353.

Piaget, J. (1972). Intellectual evolution from adolescence to adulthood. Human Development, 15, 1-12.

Piaget, J. \& Inhelder, B. (1951). La genèse de l'idée de hasard chez l'enfant. Paris: Presses Universitaires de France.

Piaget, J. \& Inhelder, B. (1963). Les opérations intellectuelles et leur développement. In P. Fraisse et J. Piaget (Eds). Traité de psychologie expérimentale, VII, L'intelligence, 109-155.

Piaget, J. \& Inhelder, B. (1966). La psychologie de l'enfant. Coll. "Que sais-je" No 369. Paris: Presses Universitaires de France. 
Protinsky, H.O. \& Hugston, G. (1980). Adolescent volume conservation abilities: a comparaison of three tests. The Journal of Psychology, 104(1), 27-30.

Ross, R.J. (1973). Some empirical parameters of formal thinking. Journal of Youth and Adolescence, 2(2), 167-177.

Schircks, A. \& Laroche, J.L. (1970). Etude des opérations intellectuelles chez des adultes de la promotion supérieure du travail. Le travail humain, 33(1-2), 99-112.

Schwebel, M. (1975). Formal operations in first-year college students. The Journal of Psychology, 91, 133-141.

Sheehan, D.J. (1970). The effectiveness of concrete and formal instructional procedures with concrete and formal operational students. Dissertation Abstracts International, $31(6-\mathrm{A}) 2748$.

Sills, T.W. \& Herron, J.D. (1976). Study of an electronic analog to the combinations of chemical bodies piagetian task. The Journal of Genetic Psychology, 129, 267-272.

Sinnot, J.D. (1975). Everyday thinking and piagetian operativity in adults. Human Development, 18, 430-443.

Somerville, S.C. (1974). The pendulum problem: Patterns of performance defining development stages. British Journal of Educational Psychology, 44(3), 266-281.

Strohm-Kitchener, K. \& King, P.M. (1981). Reflective judgment: Concepts of justification and their relationship to age and education. Journal of Applied Developmental Psychology, 2(2), 89-116 (a).

Tomlinson-Keasy, C. (1972). Formal operations in females from eleven to fifty-four years of age. Developmental Psychology, 6(2), 364.

Torkia-Lagacé, M. (1981). La pensẻe formelle chez les étudiants de collège 1: objectif ou réalité. Québec: CEGEP de Limoilou, Projet PROSIP (D.G.E.C.).

Walker, R.A., Hendrix, J.R. \& Mertens, T.R. (1979). Written piagetian task instrument: Its development and use. Science Education, 63(2), 211-220.

Wason, P.C. (1969). Regression in reasoning? British Journal of Psychology, 60, 471-480.

Wollman, W. (1977). Controlling variables: Assessing levels of understanding. Science Education, 61 (3), 371-383 (a).

Wollman, W. (1977). Controlling variables: A neo-piagetian developmental sequence. Science Education, 61 (3), 385-391 (b).

Williams, J.D., Clark, A.T. \& Brekke, B.W. (1978). Relationships among different stages of piagetian tasks with university students. In G.J. Lubin, M.K. Poulsen, J.F. Magary, M. Soto-McAlister (Eds.). Piagetian Theory and Its Implications for the Helping Professions. Proceedings Seventh Conference (Vol. II) (p. 305-313). Los Angeles, California: University of Southern California.

Nous remercions Marie-Louise Côté, Diane Dupéré, Jérôme Comtois, Diane Labre, François Lebrun, Christine Rivard, Josée Tamborini et Linda Thibault pour leur participation à la collecte des données et à l'analyse des résultats. 